\title{
Josephson soliton oscillators in a superconducting thin film resonator
}

\author{
Holm, J.; Mygind, Jesper; Pedersen, Niels Falsig; Barbara, Paola; Filatrella, G.; Davidson, A.
}

Published in:

I E E E Transactions on Applied Superconductivity

Link to article, DOI:

10.1109/77.233965

Publication date:

1993

Document Version

Publisher's PDF, also known as Version of record

Link back to DTU Orbit

Citation (APA):

Holm, J., Mygind, J., Pedersen, N. F., Barbara, P., Filatrella, G., \& Davidson, A. (1993). Josephson soliton oscillators in a superconducting thin film resonator. I E E E Transactions on Applied Superconductivity, 3(1(4)), 2504-2507. https://doi.org/10.1109/77.233965

\section{General rights}

Copyright and moral rights for the publications made accessible in the public portal are retained by the authors and/or other copyright owners and it is a condition of accessing publications that users recognise and abide by the legal requirements associated with these rights.

- Users may download and print one copy of any publication from the public portal for the purpose of private study or research.

- You may not further distribute the material or use it for any profit-making activity or commercial gain

- You may freely distribute the URL identifying the publication in the public portal 


\title{
JOSEPHSON SOLITON OSCILLATORS IN A SUPERCONDUCTING THIN FILM RESONATOR
}

\author{
J. Holm*, J. Mygind and N. F. Pedersen \\ Physics Laboratory I, The Technical University of Denmark \\ DK-2800 Lyngby \\ Paola Barbara and G. Filatrella \\ Department of Physics, University of Salerno \\ I-84100 Salerno, Italy \\ A. Davidson \\ IBM Research Division, T.J. Watson Research Center \\ Yorktown Heights, NY 10598, USA
}

\begin{abstract}
Josephson soliton oscillators integrated in a resonator consisting of two closely spaced coplanar superconducting microstrips have been investigated experimentally. Pairs of long one-dimensional Josephson Junctions with a current density about $1000 \mathrm{~A} / \mathrm{cm}^{2}$ were made in $\mathrm{Nb} / \mathrm{AlOx} / \mathrm{Nb}$ trilayer technique. Different modes of half-wave resonances in the thin-film structure impose different magnetic field configurations at the boundaries of the junctions. The dc $I V$-characteristic shows zero-field steps (ZFS) with a number of resonator-induced steps. These structures are compared to rf-induced steps generated by phase-locking of the soliton motion to an external microwave source. The two different resonant configurations identified may be interpreted as a coupling to the microwave field at the end boundary, and as a more homogeneous coupling distributed over the junction length, respectively.
\end{abstract}

\section{INTRODUCTION}

The interest in Josephson fluxon oscillators has increased because of their potential applications as electronic devices [1] such as local oscillators for SIS mixers in cryogenic integrated millimeter wave receivers for use in radio-astronomy and space-communication [2].

The one-dimensional fluxon oscillator is a Josephson tunnel junction which is much longer than the Josephson penetration depth, $\lambda_{J}$, in one direction and comparable to or less than $\lambda_{J}$ in the other direction. When the junction is properly dc biased, a localized magnetic flux quantum can oscillate back and forth in the junction and by being reflected at the edges generates electromagnetic radiation in the millimeter wave frequency range with a power in the low $\mathrm{nW}$ range. This power, however, is too low for most applications.

A possible way to raise the power without loosing the quality of the oscillator is to use one- or two-dimensional arrays of mutually phase-locked fluxon oscillators [2]. In such an array it is essential to determine the nature of the mutual coupling and to optimize the coupling between the junctions and the surrounding network. Earlier experiments showed that spontaneous phase-locking in an array

Manuscript received August 24, 1992. may take place near the resonance frequencies of a microstrip transmission line placed close to the junction [3].

Experiments with another geometry where the junctions are an integrated part of the resonator have shown mutual phase-locking [4] as well as phase-locking to an external resonator [5]. Here we report on a more detailed study of the influence of this geometry in order to better understand the nature of the interaction between the soliton and the cavity.

\section{MEASUREMENTS}

The samples were fabricated in $\mathrm{Nb} / \mathrm{AlOx} / \mathrm{Nb}$ trilayer technology with a critical current density of $10^{3} \mathrm{~A} / \mathrm{cm}^{2}$ as described elsewhere [6]. Fig. 1 gives an overview of the sample geometry. The length and width of the two Nb-film strips are $2000 \mu \mathrm{m}$ and $200 \mu \mathrm{m}$, respectively. The mutual distance is $44 \mu \mathrm{m}$. The Josephson overlap junctions (200 $\mu \mathrm{m}$ by $10 \mu \mathrm{m})$ are closely spaced near the center of one of the Nb-films. The substrate is a $625 \mu \mathrm{m}$ thick silicon chip which could be mounted onto a copper groundplane. In Fig. 1, a two-junction geometry is shown, while other samples have 4,8 or 16 junctions. The junctions are almost identical with a critical current of $10 \mathrm{~mA}$. The Josephson penetration depth is estimated to $\lambda_{J} \simeq 10 \mu \mathrm{m}$ giving a maximum plasma frequency of $130 \mathrm{GHz}$.

As evident from Fig. 1 the junctions are current biased in series, while only the sum of the voltages across the individual junctions can be measured. On samples with four junctions, the voltage could be measured over each of the two pairs. In spite of this it is without ambiguity possible to dc bias each junction independently in any point of the $I V$-characteristic [4]. The maximum voltage of the first zero-field step (ZFS1) is $100 \mu \mathrm{V}$ giving a maximum soliton oscillator frequency of $24 \mathrm{GHz}$. Fig. 2 shows the lower part of ZFS1 for one of the junctions in a four-junction sample. Also a hysteretic step at $79 \mu \mathrm{V}$, corresponding to a soliton frequency of $19 \mathrm{GHz}$, is present. A series of minor steps can also be seen on Fig. 2 at lower voltages. These self-induced substeps on the ZFS have earlier been explained as being due to a coupling between the soliton oscillator and an external resonator $[5,7]$. By applying a small magnetic field in the plane of the junction it was possible to marginally change the boundary condi-

$1051-8223 / 93 \$ 03.00 \odot 1993$ IEEE 


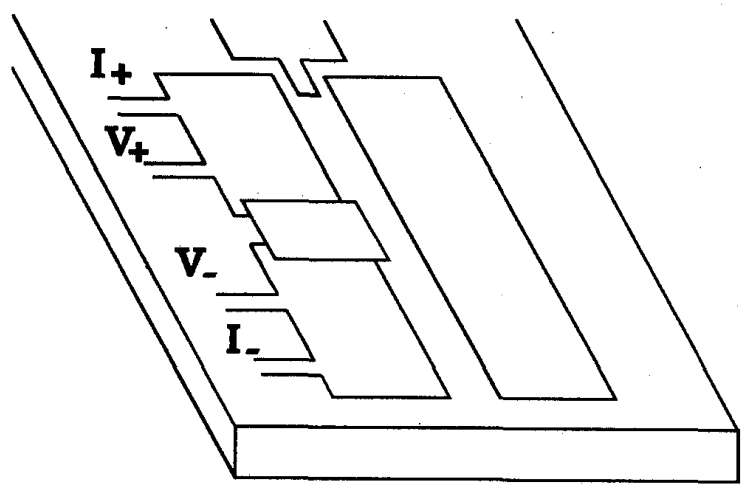

Fig. 1 Schematic drawing of the sample geometry. The loosely coupled microstrip antenna shown in the top of the figure is used for the microwave measurements.

tions for the reflection of the solitons, which in turn changed the coupling. The substeps were approximately one order of magnitude more sensitive to the magnetic field than the critical current and, actually, some substeps could only be seen with an applied magnetic field.

The hysteretic step contains regions where the differential resistance $R_{D}=\partial V_{d c} / \partial I_{d c}$ is very low compared to that of the ZFS. This is important for applications of the soliton oscillator as a narrow linewidth microwave oscillator, because as a simple model has earlier suggested [8], the linewidth of the emitted radiation $\Delta \nu_{M}$ from a soliton oscillator is proportional to $R_{D}^{2} / R_{S}$, where $R_{S}=V_{d c} / I_{d c}$ is the static resistance of the bias point. On the hysteretic step strong microwave radiation emission corresponding to the soliton frequency was detected. Detailed measurements of the linewidth showed that the above mentioned proportionality actually was fulfilled, indicating that low amplitude Nyquist thermal noise dominates the voltage fluctuations in the junction.

\section{RESONANT MODES}

From earlier investigations [7] it is known that the present geometry can support several resonant modes. One is a coplanar mode, where the rf electric and magnetic fields are mainly confined to the narrow gap between the Nb-films. This mode preferably couples to the Josephson junction boundary facing the gap.

The others are the microstrip modes supported by each of the $\mathrm{Nb}$-films and the groundplane placed beneath the substrate. The fields are here more evenly distributed across the films. The latter modes have slightly different resonance frequencies because the symmetry is broken by the presence of junctions in one of the $\mathrm{Nb}$-films.

To clarify the problem with coupling to several coexisting resonance modes, we systematically tried to suppress each of the modes by changing the geometry. The effect on the substeps of removing the groundplane was negligible, while measurements on a geometry where the

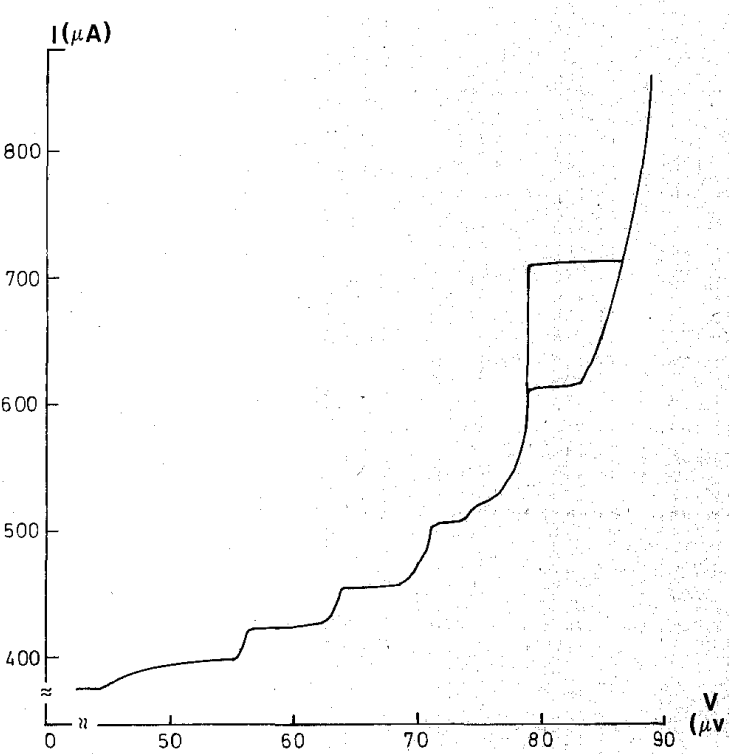

Fig. 2 Lower part of ZFS1 for a four-junction sample (U1-4JJ). The hysteretic step is at $79 \mu \mathrm{V}$. No groundplane was present. $T=4.2 \mathrm{~K}$.

adjacent $\mathrm{Nb}$-film was missing, did not reveal any substeps at all. From this it is inferred that the substeps on the ZFS are due to coupling between the soliton oseillator and the coplanar resonance mode.

In order to model the geometry used in Ref. 2 , where a series-biased array of long overlap junctions was located near one end of a $50 \Omega$ microstrip half-wave resonator, Monaco et al. [3] developed a simple analytical approach with overlap junctions coupled to a lumped element linear tank circuit. This model predicts locking between the soliton oscillators and the tank circuit, when the junction is operated near the fundamental resonance frequency or its subharmonics [9]. The height of these subharmonic steps was seen to vary approximately inversely with the subharmonic number.

In contrast to the lumped element circuit, the distributed resonator discussed here exhibits resonances at all multiple $n$ (integer) of one half-wavelength and gives rise to locking whenever the soliton frequency $\nu_{s}$ equals a fractional $m$ (also integer) harmonic $(n / m) \nu_{c}$ of the half wavelength resonance frequency of the coplanar mode $\nu_{c}$. Indeed, the positions of the steps in Fig. 2 correspond very well to $n / m=2 / 5$ and $n / m=4 / 9$, assuming that the hysteretic step corresponds to $n / m=1 / 2$.

At lower temperatures, the substeps became larger, while at sufficiently high temperatures the substeps completely disappeared. When irradiating the junction at this high temperature with microwaves via the microstrip antenna shown at the top of Fig. 1, rf-induced phase-locked steps were observed on the ZFS1 in agreement with the relation [10]: 


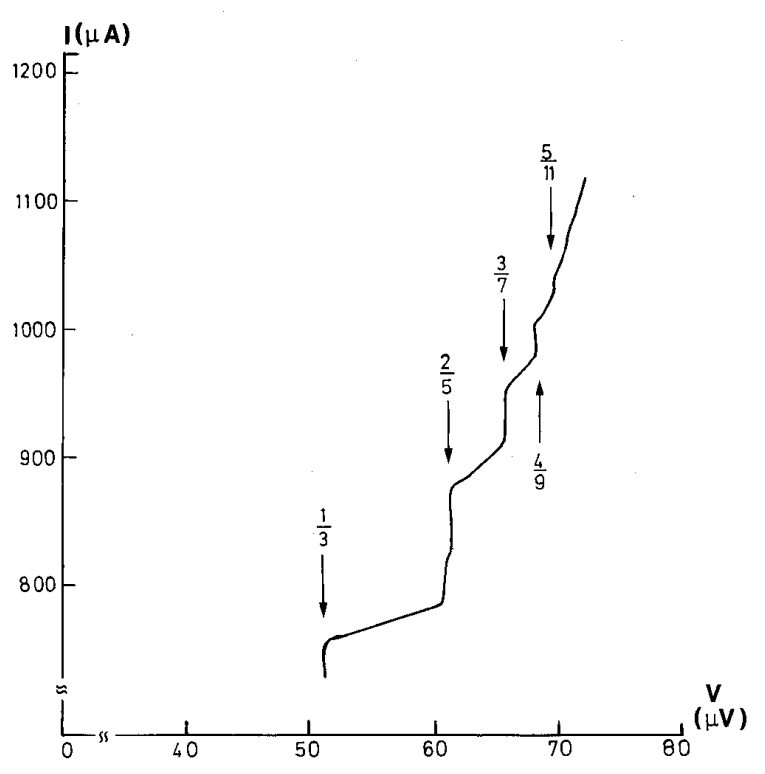

Fig. 3 Lower part of the same ZFS1 as in Fig. 2, but at higher temperature (see text), and under influence of an external microwave field at $37.2 \mathrm{GHz}$. The $I V$-curve shows fractional harmonic locking of the soliton oscillator to the external source. Arrows indicate $n / m$ (see text).

$$
\nu_{s}=\frac{n}{m} \nu_{r f}
$$

where $\nu_{r f}$ is the frequency of the applied microwave field. A typical $I V$-curve (Fig. 3) shows the well-known structure of the devil's staircase with locking at $n / m=\{1 / 3$, $2 / 5,3 / 7,4 / 9,5 / 11, \ldots, 1 / 2\}$. In Fig. 2 , the $3 / 7$ and $5 / 11$ harmonics are missing. However, by applying a small external magnetic field these steps actually appeared on the ZFS.

Also samples with a single pair of junctions were investigated. Several substeps were seen, and again their positions correspond to locking at fractional harmonics of the resonance frequency of the coplanar mode. Besides reproducing the substeps below $n / m=1 / 2$, the symmetry around $1 / 2$ was confirmed by the following sequence of observed substeps at higher voltages: $n / m=$ $\{1 / 2,6 / 11,5 / 9,4 / 7,3 / 5\}$. For even $n$ it was necessary to apply a small magnetic field in order to observe the substeps.

The rf-driven long Josephson junctions show constant-voltage current steps, which has been attributed to the phase-locking of single fluxon oscillations to the external signal. The main problem in modelling this locking is to choose the correct boundary conditions, i.e., whether it is more realistic to model the interaction between the junction and the microwave field by introduc- ing time-dependent boundary conditions representing the rf magnetic field or by adding an rf-current term to the perturbed sine-Gordon equation, used to model the dynamics of the junction [9]. In the former approach, known as the "M-model", the energy exchange takes place only at the boundaries. In the latter, known as the "E-model", the energy is uniformly supplied along the junction [9]. Ref. 11 suggests an experimental test which may discriminate between these types of couplings in the small signal limit. Assuming pure magnetic coupling, the phase-locked step is symmetrical relative to the unperturbed ZFS. With both coupling effects present, the uniform coupling may shift the rf-steps towards a higher bias value so that the resulting rf-steps become asymmetrical [11].

By operating the junction as a self-detector. i.e. current biasing it at the superconducting gap and measuring the small changes in voltage when applying a microwave signal, it was possible to detect resonances in the system when the frequency of the signal was varied. In a twojunction sample such measurements combined with measurements of the height of the rf-induced steps and the suppression of critical current, both as function of signal frequency, revealed the presence of two resonances at $\nu_{r f}=14.5 \mathrm{GHz}$ and at $\nu_{r f}=17.8 \mathrm{GHz}$, respectively. The latter is close to the hysteretic substep at $18 \mathrm{GHz}$ ascribed to the coplanar mode in this sample. Experiments on other two-junction samples without groundplane did not show any resonant behaviour around $15 \mathrm{GHz}$, substantiating that the first resonance is due to the microstrip mode. Fig. 4 shows the height $\Delta \mathrm{I}$ of the phase-locked step at these frequencies as function of the applied signal amplitude found as the square root of the relative power read on a calibrated attenuator inserted between the millimeter wave source and the Josephson junction. The step at 14.5 $\mathrm{GHz}$ is nearly symmetric relative to the unperturbed ZFS, while the other at $17.8 \mathrm{GHz}$ is shifted upwards. A similar variation was actually seen in Ref. 7 . It is therefore experimentally possible to distinguish between different kinds of coupling between the soliton oscillator and the external field.

\section{CONCLUSION}

In a coplanar thin-film structure with integrated pairs of long Josephson junctions locking behaviour between distributed resonator modes and the soliton oscillator has been observed. Detailed investigations reveal that the soliton oscillator preferably locks to the coplanar mode with the strongest coupling to one of the end boundaries. By comparing to measurements on rf-induced steps, it was seen that the positions of the self-induced substeps followed a devil's staircase. The magnitude of the substeps was very sensitive to an applied dc magnetic field.

\section{ACKNOWLEDGEMENTS}

Fruitful discussions with G. Costabile, N. GrønbechJensen, R.D. Parmentier, and M.R. Samuelsen, are acknowledged. This work was partially conducted under the auspices of the Consortium for Superconducting Electronics (CSE), with support from the Defence Advanced Research Projects Agency (Contract Number MDA972- 
90-C-0021).
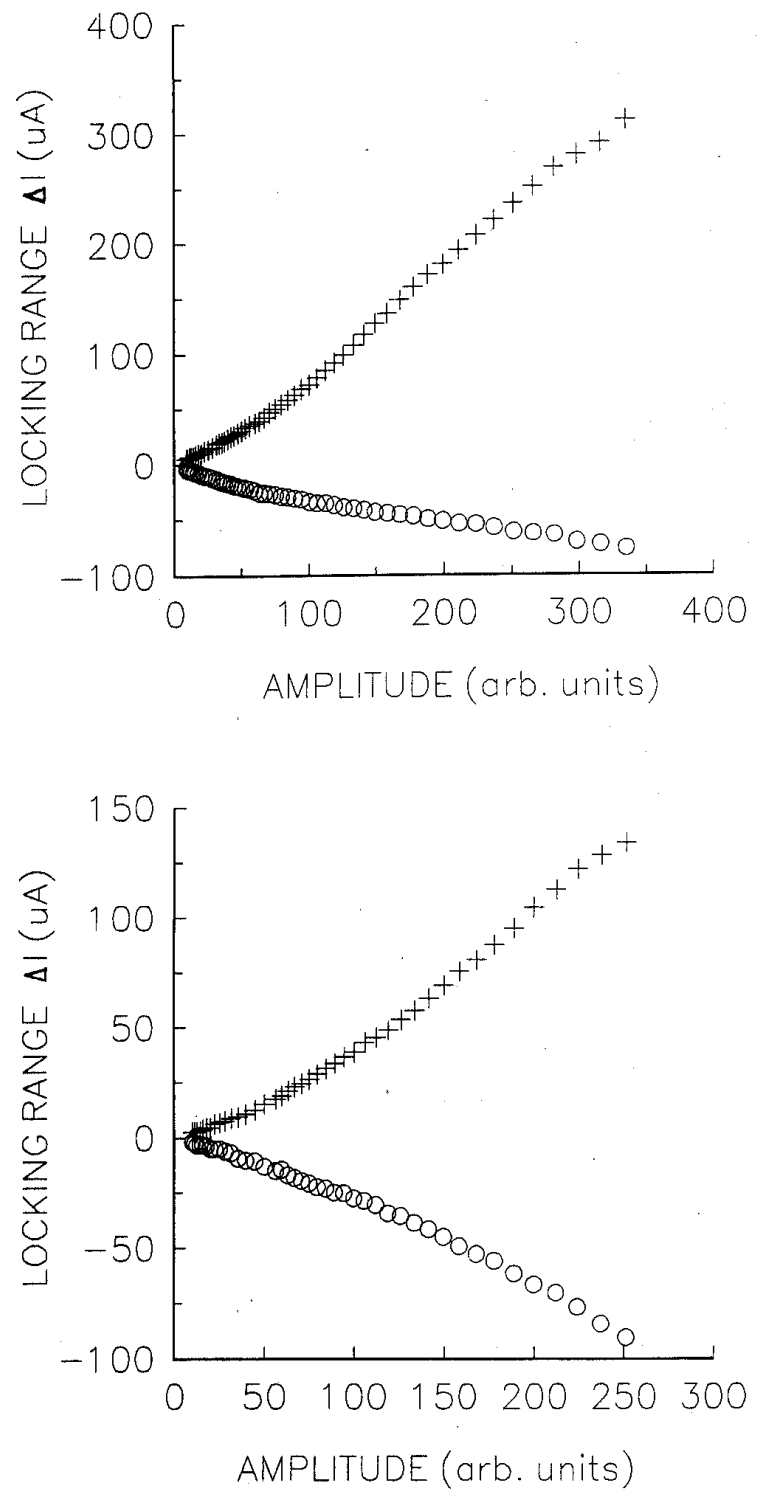

Fig. 4 Dependence of locking range $\Delta I$ of the rf-induced step on applied microwave signal in a two-junction sample with groundplane. The upper and lower marks indicate the bias current at which phaselocking is lost. (a) $\nu_{r f}=17.8 \mathrm{GHz}$. (b) $\nu_{r f}=14.5$ $\mathrm{GHz}$. In both cases the external driving frequency equals the soliton frequency. Zero current corresponds to the bias point of the unperturbed ZFS.

\section{REFERENCES}

* Also at: Danish Institute of Fundamental Metrology, B 309, Lundtoftevej 100, DK-2800 Lyngby

[1] N. F. Pedersen, "Fluxon Electronic Devices," IEEE Trans. Magn, MAG-27, 3328 (1991).

[2] S. Pagano, R. Monaco and G. Costabile, "Microwave Oscillator Using Arrays of Long Josephson Junctions," IEEE Trans. Magn. MAG-25, 1080 (1989).

[3] R. Monaco, N. Grønbech-Jensen and R.D. Parmentier, "Self-locking of fluxon oscillators in series arrays of niobium Josephson tunnel junctions coupled to a linear resonator," Phys. Lett. A 151, 195 (1990).

[4] A. Davidson and N.F. Pedersen, "Experiments on the interaction between long Josephson junctions and a Coplanar strip resonator," Appl. Phys. Lett 60, 2017 (1992).

[5] A. Davidson, N. Grønbech-Jensen and N.F. Pedersen "Josephson soliton Oscillator arrays for SIS mixers," IEEE Trans. Magn., MAG-27, 3347 (1991).

[6] M. B. Ketchen, D. Pearson, A.W. Kleinsasser, C.K. Hu, M. Smyth, J. Logan, K. Stawiasz, E. Baran, M. Jaso, T. Ross, K. Petrillo, M. Manny, S. Basavaiah, S. Brodsky, S.B. Kaplan, W.J. Gallagher and M. Bhushan, "Sub- $\mu \mathrm{m}$, planarized, Nb-AlOx-Nb Josephson process for $125 \mathrm{~mm}$ wafers developed in partnership with Si technology," Appl. Phys. Lett. 59, 2609 (1991).

[7] Paola Barbara, A. Davidson, G. Filatrella, J. Holm, J. Mygind and N.F. Pedersen, "Coupling of a Josephson soliton Oscillator to coplanar and microstrip cavities," Phys. Lett. A 165, 241 (1992).

[8] J. Bindslev Hansen, Yu.Ya. Divin, J. Mygind and M.R. Samuelsen, "Measurements of frequency and linewidth of the radiation emitted from overlap Josephson junctions," in $S Q U I D-85$, H.D. Hahlbohm and H. Lübbing, Eds. W. de Gruyer \& Co., Berlin, 1985, pp. 489-494.

[9] G. Filatrella, N. Grønbech-Jensen, R. Monaco, S. Pagano, R.D. Parmentier, N.F. Pedersen, G. Rotoli, M. Salerno, and M.R. Samuelsen in: Nonlinear Superconductive Electronics and Josephson devices, Edited by G. Costabile, S. Pagano, N.F. Pedersen, and M. Russo, Plenum Press, New York, 1991, pp 253-269.

[10] M. Scheuermann, J.T. Chen and Jhy Juin Chang, "Interaction between microwaves and a single vortex in a long Josephson tunnel junction, J. Appl. Phys. 54, 3286 (1983).

[11] J.C. Fernandez, G. Filatrella and G. Reinisch, "Comparision between electric and magnetic rf-drive in long Josephson junctions," Phys. Lett. A 153, 446 (1991). 